\title{
Rumination, Mood and Cognitive Performance
}

\author{
Jay K. Brinker, Mel Campisi, Laura Gibbs, Rebekah Izzard \\ Research School of Psychology, The Australian National University, Canberra, Australia \\ Email: jay.brinker@anu.edu.au \\ Received October $29^{\text {th }}, 2012$; revised December $10^{\text {th }}, 2012$; accepted January $9^{\text {th }}, 2013$
}

\begin{abstract}
Rumination, DEFINED AS REPETITIVE, RECURRENT AND UNCONTROLLABLE THINKING, has been implicated in cognitive impairments, particularly dysexecutive function, in people with depressed mood, what is now critical is an examination of the link between rumination and cognitive impairment independent of mood. Rumination's direct relationship to basic cognitive functions may help explain the stability of rumination, and how it predicts future episodes of depressed mood. Two experiments examined how trait and experimentally induced rumination, and negative mood relate to attention and inhibition at varying levels of cognitive load in non-depressed participants. Study 1 found trait rumination significantly predicted errors of attention when cognitive load was low or high, but not at a medium level. Trait rumination also interacted with frequency of task unrelated thoughts to predict performance. Negative mood significantly predicted errors of inhibition also when the cognitive load was low or high. Study 2 expanded on the first by including two additional measures of executive function. Results were replicated for the first study and showed a ruminative thought style predicted inhibition, reaction time and set shifting in the additional measures.
\end{abstract}

Keywords: Rumination; Mood; Executive Functions; Cognitive Load; Off-Task Thoughts

\section{Introduction}

There are many ways to conceptualise rumination; a response to depressed mood (Nolen-Hoeksema, Parker, \& Larson, 1994; Verhaeghen, Joorman, \& Khan, 2005), an emotion regulation strategy (Nolen-Hoeksema, Wisco, \& Lyubomirsky, 2008), a form of self-focused attention (Mor \& Winquist, 2002), as a process or style of thinking (Brinker \& Dozois, 2009) and so on. These conceptions are not necessarily contradictory, but simply different ways of considering and examining a complex phenomenon. In its most general sense, rumination is a style of thinking characterised by repetitive, recurrent, intrusive and uncontrollable thoughts (Brinker \& Dozois, 2009). The relationship between rumination and cognitive impairment has been demonstrated, but what is not known is 1) the relationship between rumination and cognition independent of depressed mood; and 2) examination of the causal relationship between rumination and impairment. These may be addressed by measuring rumination as an individual difference in a non-depressed sample using a measure of the process of rumination, and by experimentally manipulating rumination to observe changes in cognitive performance.

Within the different conceptions of rumination, it is generally agreed that rumination is a stable phenomenon (Brinker \& Dozois, 2009; Nolen-Hoeksema, Wisco, \& Lybomirsky, 2008) with serious clinical implications due to the consistent relationship with depressed mood. Rumination is related to greater depressed mood, longer depressive episodes and the occurrence of future depressive episodes (Brinker \& Dozois, 2009; NolenHoeksema, Wisco, \& Lybomirsky, 2008). The link to future episodes of depression raises two important points. First, even when negative mood is ameliorated, the process of thinking in a ruminative style may continue, and the vulnerability posed by rumination may continue. Second, the stability of ruminative thinking suggests the process may be related to individual differences in basic cognitive function-in particular executive functioning.

Executive functions are a group of cognitive abilities that enable goal directed behaviour including attentional control, inhibitory systems and cognitive flexibility. Regardless of content, ruminative thoughts reflect an unintentional shift in attention away from the task at hand (Martin \& Tesser, 1996), and the failure of cognitive inhibitory systems to limit attention to task-relevant information (Carson, Peterson, \& Higgins, 2003; Hertel, 1997; Joormann, 2004). In other words people who ruminate are unable to inhibit the ruminative thoughts and maintain attention elsewhere.

Research has demonstrated a relationship between rumination and attentional inflexibility (Davis \& Nolen-Hoeksema, 2000; Lo, Lau, Cheung, \& Allen, 2012). Watkins and Brown (2002) suggest that rumination reduces cognitive flexibility by overloading cognitive resources, primarily in the form of deficits in switching and inhibition (Miyake et al., 2000). Whitmer and Banich (2007) suggest that switching attention from one conceptual set to another is difficult for individuals who ruminate and show rumination is linked to difficulties with inhibiting previously relevant task sets in a non-depressed sample. Davis and Nolen-Hoeksema (2000) also found evidence for attentional inflexibility, with ruminators making more perseveration errors on the Wisconsin Card Sorting Test and taking more time on a measure of psychomotor speed compared to non-ruminators.

As mentioned, Watkins and Brown (2002) argue performance impairment may result from limited cognitive resources, and rumination may further consume resources that would otherwise be directed towards the primary task. Increasing the cognitive demands of the current external task has been found to lead to a reduction in the frequency of off-task thoughts (e.g. 
Teasdale et al., 1995). Antrobus (1968) observed that increasing the speed of stimulus presentation in a signal-detection task increased the cognitive demands for processing task related information, resulting in an improvement in task performance. This was attributed to more cognitive resources needing to be directed towards the task, leaving fewer cognitive resources available for the production of off-task thoughts. These results suggest that attention-demanding tasks may disrupt rumination (Teasdale et al., 1995). Lyubomirsky and colleagues (2003) tested the effect of dysphoric rumination on participant's performance on three academic tasks (reading a passage, watching a videotaped lecture and proofreading written text) and found that decrements in task performance tended to be greater for tasks with mild to moderate difficulty. Lyubomirsky and colleagues (2003) also found that dysphoric participants who ruminated were slower at the tasks than the other comparison groups even if their accuracy was equivalent.

Typically, rumination is tested in participants experiencing dysphoric mood (natural or induced) with much research suggesting it is the combination of negative mood and rumination that produces effects (Lyubomirsky, Kasri, \& Zehm, 2003). However, there is reason to suppose that a ruminative style of thinking may be related to impaired executive functions regardless of mood. The previous research examining intrusive thoughts, inhibition and executive function in relation to cognitive performance illustrates differences in non-depressed samples without inducing negative mood (Carson, Peterson, \& Higgins, 2003, Teasdale et al., 1995, Whitmer \& Banich, 2007). Rumination has been linked to executive function (Davis \& Nolen-Hoeksema, 2000) and Williams, Suchy and Rau (2009) argue that there are individual differences in executive function both in conjunction with and independent of mood. Given the firm basis of research in rumination and depression combined, it is now time to examine the influence of rumination in a non-depressed sample without inducing negative mood. It is hypothesised that rumination, even in the absence of depressed mood, will be related to impairment in executive function.

\section{Study 1}

Several variables are involved when considering the above findings; attention, inhibition, cognitive load, task unrelated thoughts and both trait and induced rumination. This project will examine these variables together by employing a continuous performance task to directly manipulate cognitive load and measure attention and inhibition. Further, a baseline measure of trait rumination and experimentally induced rumination will be included. It is hypothesised that participants in the rumination condition will show poorer performance than those in a distraction condition, on attention and inhibition when cognitive load is low. It is also predicted that participants high on trait rumination will perform more poorly than those with low trait rumination. Finally, greater frequency of off-task thoughts will be related to poorer cognitive performance.

\section{Method}

\section{Participants}

One-hundred first year psychology students (31 male and 69 female) from the Australian National University participated in the study ranging in age from $17-57$ years $(\mathrm{M}=19.83, \mathrm{SD}=$ 4.77). Participants WERE RECRUITED VIA POSTERS
PLACED AROUND THE DEPARTMENT AND ON THE FIRST YEAR COURSE WEBSITE. THEY received one hour of course credit for their participation. THERE WAS NO SET INCLUSION OR EXCLUSION CRITERIA AND PARTICIPANTS WERE NOT RECRUITED BASED ON PREVIOUS MENTAL HEALTH HISTORY.

\section{Materials}

Positive and Negative Affect Schedule (PANAS-X; Watson \& Clark, 1994): THIS MEASURE WAS INCLUDED TO CONTROL FOR BASELINE MOOD. The PANAS consists of 20 mood adjectives: 10 positive (PANAS-P) and 10 negative (PANAS-N). Respondents are asked to rate on a 5-point scale the degree to which they have experienced each of these moods in the past 24 hours. The scale ranges from 1 (not at all) to 5 (very much). These scales were used as a baseline measure of the degree of positive and negative affect participants were experiencing prior to completing the study. The Chronbach's alphas for this study were .87 for the PANAS-P and .85 for the PANAS-N.

Ruminative Thought Styles Questionnaire (RTS; Brinker \& Dozois, 2009). The RTS is a measure of a general ruminative thought style characterised by repetitive, recurrent, intrusive and uncontrollable thinking. It consists of 20 statements that respondents rate as self descriptive or not on a Likert-type scale from 1 -not at all like me to 7-very much like me. The total scores range from 20 to 140 with higher scores indicating greater ruminative thinking. The RTS shows good internal reliability ( $\alpha=.87$ to .92$)$, good test-retest reliability $(r=.80)$ and good convergent and divergent validity (Brinker \& Dozois, 2009). Cronbach's alpha for the current study is .91.

Rumination/Distraction Induction (Lyubomirsky \& NolenHoeksema, 1993): This task IS THE EXPERIMENTAL MANIPULATION AND was designed to induce either a ruminative or distractive style of thought in participants, and has been widely used in rumination research (e.g. Rimes \& Watkins, 2005; Watkins \& Moulds, 2005; Lyubomirsky, Kasri, \& Zehm, 2003). The rumination condition asks participants to think about a series of 45 self-focused statements, (e.g. "think about what your feelings might mean"), and is designed to focus participants on the meaning, causes and consequences of their current feelings (Nolen-Hoeksema, Wisco, \& Lyubomirsky, 2008). The distraction condition required participants to focus on 45 statements designed to shift participants' focus away from themselves, (e.g. "think about a boat sailing on the ocean"). Each induction lasted for eight minutes. While these are typically used with individuals currently experiencing depressed mood they may be of use with a healthy sample by inducing self-focused attention, a component of the ruminative response style.

Continuous Performance Task (CPT): THIS TASK MEASURES ATTENTION AND INHIBITION-THE DEPENDENT VARIABLE. Participants completed a computer task involving attending to a series of letters presented on the screen and responding by pressing the space bar whenever an " $\mathrm{X}$ " was presented. Letters were presented in nine blocks of 20 letters, five of which were " $\mathrm{X}$ "s in each block. All letters were displayed for $250 \mathrm{~ms}$ each. The inter-stimulus intervals were 1, 2 and 4 seconds (fast, medium and slow speeds, respectively) creating 3 levels of cognitive load-high, medium and low. Participants viewed three blocks for each of the speeds. Participants were also given a 'practice round' before commencing 
the task. The task took approximately eight minutes to complete, and produced two outcome measures for each of the speeds: number of correct hits (attention) and number of false hits (failure to inhibit). Because the key comparison is across experimental groups, not across speed blocks, the order of presentation speed was held constant for all participants.

Off-Task Thoughts: Following the CPT, participants reported the type and frequency of off-task thoughts they experienced AS A POSSIBLE MEDIATING VARIABLE. Participants reported frequency of task related and unrelated thoughts, and frequency of repeating thoughts using Likert scales ranging from 1 (not at all) to 7 (frequently).

Mood Rating Scale: A visual analogue scale (VAS) was included as a brief, repeatable mood measure to assess changes in participant's mood throughout the study. Based on Lyubomirsky \& Nolen-Hoeksema's (1995) measure, participants were asked to indicate the degree of positive or negative affect they were currently experiencing by moving a marker up or down a $100 \mathrm{~mm}$ line representing a continuum from 0 (extremely negative affect) to 100 (extremely positive affect). Higher scores on these reflect lower negative mood.

\section{Procedure}

Participants completed the study in a computer lab in groups of $5-10$ and were randomly assigned to either the rumination or distraction condition. All sections of the study were presented and completed by participants on computer, and participants simply clicked on the "Next" button to move through the protocol. Participants first completed the PANAS-X and the first visual analogue scale mood measure (Mood 1) to assess their baseline mood, and the RTS to assess their trait ruminative thinking style. THESE VARIABLES ACT AS CONTROL WHEN EXAMINING THE EFFECT OF THE EXPERIMENTAL MANIPULATION. They were then presented with their assigned induction procedure. Participants were asked to rate their current mood using a second visual analogue scale (Mood 2) and then complete the Continuous Performance Task. Participants reported the off-task thoughts they experienced during the CPT and completed a final VAS (Mood 3).

\section{Results and Discussion}

T-tests revealed no significant between group differences for age $(\mathrm{t}=-1.46, p=.15)$ or mood as measured by the PANAS (positive $\operatorname{mood} \mathrm{t}=-.70, p=.49$; negative $\operatorname{mood} \mathrm{t}=1.62, p$ $=.11)$ and VAS1 prior to the induction $(\mathrm{t}=-.87, p=.34)$. However, despite participants being randomly assigned to conditions, there was a significant difference between experimental groups on baseline ruminative thought styles $(\mathrm{t}=2.23, p<.05)$ with the rumination group reporting higher levels of trait ruminative thought than the distraction group $(\mathrm{M}=91.06$ and 83.24 respectively). Because of this, analyses examining the RTS will be interpreted with caution.

Correlations were conducted to examine the relationships between baseline measures prior to the inductions (Table 1). All correlations were significant and in the expected direction (Papageorgiou \& Siegle, 2003) with GREATER rumination being significantly related to greater negative mood as measured by the PANAS-N and the visual analogue scales.

Group comparisons on post induction differences in mood show the inductions produced a difference in mood between the groups that was near to significance $(\mathrm{t}=1.946, p=.06)$. Be- cause of the imbalance in RTS scores across the experimental groups, with the rumination group having higher RTS scores, this difference in post-induction mood may be an artifact of assignment bias due to the high correlation between ruminative thought styles and negative mood. Hierarchical linear regression examined the prediction of post induction mood, with baseline mood entered in the first step, and induction condition and trait rumination entered into the second step. Only baseline $\operatorname{mood}(\beta=-.223, p=.03)$ and ruminative thought style $(\beta=$ $-.507, p=.000)$ were significant predictors.

Linear regression analyses predicted performance on the six CPT variables (i.e. correct and false hits for each of the 3 levels of cognitive load). Baseline mood was entered into the first step, with RTS score and induction condition entered in the second. For correct hits, as a measure of attention, RTS score was a significant predictor at both the high and low cognitive load but not the medium (Table 2). Mood was a significant predictor of

Table 1.

Baseline correlations.

\begin{tabular}{ccccc}
\hline & PANAS-N & Mood 1 & M & SD \\
\hline RTS & $.540^{* *}$ & $-.604^{* *}$ & 87.15 & 17.91 \\
PANAS-N & & $-.444^{* *}$ & 20.47 & 7.05 \\
Mood 1 & & & .673 & .208 \\
\hline
\end{tabular}

Note: ${ }^{* *} p=.01$.

Table 2.

Predicting cognitive performance by degree of cognitive load controlling for baseline mood.

\begin{tabular}{lccccccc}
\hline & \multicolumn{6}{c}{ False Hits (Inhibition) } & \multicolumn{3}{c}{ Correct Hits (Attention) } \\
\hline High Load & B & SE B & $\beta$ & B & SE B & $\beta$ \\
Step 1 & & & & & & \\
\multicolumn{1}{c}{ Mood } & .034 & .009 & .362 & -.040 & .008 & $-.444^{* *}$ \\
Step 2 & & & & & & \\
$\quad$ Mood & .031 & .011 & $.329^{* *}$ & -.013 & .008 & -.147 \\
$\quad$ RTS Score & .003 & .004 & .085 & -.021 & .003 & $-.575^{* *}$ \\
$\quad$ Condition & .113 & .129 & .085 & -.110 & .101 & -.086 \\
$\quad$ Medium Load & & & & & & \\
Step 1 & & & & & & \\
$\quad$ Mood & .001 & .001 & .036 & .000 & .007 & -.002 \\
Step 2 & & & & & & \\
$\quad$ Mood & -.001 & .002 & -.038 & .004 & .009 & .053 \\
$\quad$ RTS Score & .001 & .001 & .115 & -.004 & .003 & -.149 \\
$\quad$ Condition & -.016 & .021 & -.081 & -.165 & .105 & -.161 \\
$\quad$ Low Load & & & & & & \\
Step 1 & & & & & & \\
$\quad$ Mood & .086 & .012 & $.600^{* *}$ & -.090 & .015 & $-.514^{* *}$ \\
Step 2 & & & & & & \\
$\quad$ Mood & .093 & .014 & $.649^{* *}$ & -.029 & .014 & $-.168^{*}$ \\
$\quad$ RTS Score & -.006 & .005 & -.106 & -.044 & .006 & $-.644^{* *}$ \\
$\quad$ Condition & -.105 & .166 & -.052 & -.011 & .171 & -.005 \\
\hline
\end{tabular}

Note: ${ }^{*} p<.05 ;{ }^{* * *} p<.01$. 
correct hits, but only when cognitive load was low. For false hits, as a measure of failed inhibition, only baseline mood was a significant predictor, but again, only at high or low cognitive load (Table 2).

In relation to inhibition, it appears that mood alone accounts for variance in scores at fast and slow speeds. This supports the previous literature showing that negative mood is related to cognitive deficits (Austin, 2001). In particular, Koster, De Lissnyder, Derakshan and De Raedt (2011) propose the impaired disengagement hypothesis as a comprehensive explanation of the neurocognitive basis of these findings. This theory suggests that individuals encounter some stressor that prompts self-reflective/negative thinking.

Some individuals are unable to disengage from this negative thinking leading to prolonged rumination and subsequently task impairments and negative mood. This model is circular with negative mood feeding back into the loop at the point where the person fails to disengage from the self-reflective/negative thinking. The current findings linking negative mood to reduced ability to inhibit responses, may be one mechanism by which this happens - these individuals are unable to inhibit the self-reflective/negative thoughts. Disengagement may be further hampered by the person's inability to maintain attention elsewhere due to the ruminative style of the self-reflective/ negative thinking.

Scores on the ruminative thought styles scale predicted errors of attention after controlling for negative mood. It appears that mood and ruminative processes each relate to a different impairment in abilities needed to disengage from maladaptive thoughts and engage their attention elsewhere. The common cooccurrence of rumination and mood may be a particularly powerful vulnerability.

Following the continuous performance task, participants were asked to report the frequency of off-task thoughts in general, off-task thoughts directly related to the induction procedure and frequency of repeating thoughts. The two induction groups did not significantly differ in the self-reported frequency of these thoughts. However, the RTS significantly predicted general off-task thought frequency, even when controlling for baseline negative mood and induction condition $(\beta=.241, p$ $=.05$ ). Hierarchical regressions were used to further explore the predictive ability of ruminative thought styles and off-task thoughts on task performance.

Baseline mood was entered into the first step, the main effects of RTS score and frequency of off-task thoughts were entered into the second step, and the interaction of these two variables was entered into the third (Table 3). Ruminative thought style and frequency of off-task thoughts interacted to significantly predict errors of attention when cognitive load was high or low. As suggested by previous research, increasing cognitive demands may improve performance by preventing the intrusion of off-task thoughts, but there may be a point where the demands are too great and a decrement in performance is seen (Lyubomirsky et al., 2003; Teasdale et al., 1995). These results suggest it is the combination of a ruminative style of thinking and the experience of off-task thoughts that predicts impaired performance.

The results of this study are very interesting and speak to the possible differential effects of a ruminative thought style and negative mood. The separation of these two variables may be artificial because of their high co-occurrence, but recognition of this divide may have important consequences for treatment.
Table 3.

Rumination and off task thoughts predicting attention.

\begin{tabular}{|c|c|c|c|}
\hline \multirow[b]{2}{*}{ High Load } & \multicolumn{3}{|c|}{ Correct Hits (Attention) } \\
\hline & $\mathrm{B}$ & SE B & $\beta$ \\
\hline \multicolumn{4}{|l|}{ Step 1} \\
\hline Baseline Mood & -.040 & .008 & $-.444^{* *}$ \\
\hline \multicolumn{4}{|l|}{ Step 2} \\
\hline Baseline Mood & .031 & .011 & $.329^{* *}$ \\
\hline RTS Score & .003 & .004 & .085 \\
\hline Frequency of Off Task Thoughts & .113 & .129 & .085 \\
\hline \multicolumn{4}{|l|}{ Step 3} \\
\hline Baseline Mood & -.010 & .008 & -.113 \\
\hline RTS Score & -.003 & .008 & -.081 \\
\hline Frequency Off Task Thoughts & .427 & .199 & $.774^{*}$ \\
\hline RTS $\times$ Frequency Off Task Thoughts & -.005 & .002 & $-1.051^{*}$ \\
\hline \multicolumn{4}{|l|}{ Low Load } \\
\hline \multicolumn{4}{|l|}{ Step 1} \\
\hline Mood & .086 & .012 & $.600^{* *}$ \\
\hline \multicolumn{4}{|l|}{ Step 2} \\
\hline Mood & .093 & .014 & $.649^{* *}$ \\
\hline RTS Score & -.006 & .005 & -.106 \\
\hline Condition & -.105 & .166 & -.052 \\
\hline \multicolumn{4}{|l|}{ Step 3} \\
\hline Baseline Mood & -.022 & .013 & -.126 \\
\hline RTS Score & .001 & .013 & .013 \\
\hline Frequency Off Task Thoughts & 1.157 & .321 & $1.085^{* *}$ \\
\hline RTS $\times$ Frequency Off Task Thoughts & -.014 & .004 & $-1.454^{* *}$ \\
\hline
\end{tabular}

Note: ${ }^{*} p<.05 ;{ }^{* *} p<.01$.

While depressed mood may be ameliorated by treatment, the lingering ruminative thinking style may remain as the link to future episodes of depressed mood (Brinker \& Dozois, 2009). Trait rumination predicted frequency of off-task thoughts and the interaction of these predicted impaired performance in the absence of depressed mood. This speaks to the importance of stable ruminative thinking styles occurring in everyday life and the impact it may have on cognitive processes. However, the inequity between groups on RTS scores requires confirmation with a second sample to confidently draw conclusions.

\section{Study 2}

The unexpected group differences in RTS scores in the first sample need to be addressed to confirm the findings from study 1 and provide a clearer understanding of how the constructs of ruminative thought style, mood and cognitive performance are related. This second study replicates the first but with the exclusion of the unrelated thoughts measure and the addition of two new measures of cognitive flexibility-The Wisconsin Cart Sorting Task, and the Simon Task. The Wisconsin Card Sorting Task (WCST) is a measure of perseveration - a presentation of the inability to shift set. This task was designed to assess brain pathology and therefore may not be sensitive enough to assess 
individual differences in a healthy population. Given this potential limitation, the Simon Task, a computer based measure of inhibition based on the Stroop task, was included having been successfully used in analogue studies using non-impaired samples (Simon, 2010).

\section{Method}

\section{Participants}

One hundred and twenty eight participants completed the current study. The majority of these participants were first year psychology students at the Australian National University participating as a course requirement. The remaining participants included senior level psychology student volunteers and volunteer community members. The study included $65 \%$ females and $35 \%$ males, with an age range of $18-62(\mathrm{M}=22.3, \mathrm{SD}=9.54)$; $58 \%$ were Caucasian.

\section{Materials}

In addition to the materials repeated from study one, this study includes.

Wisconsin Card Sorting Task (WCST; Grant \& Berg, 1948). Grant and Berg (1948) designed this task as a measure of set shifting ability. Participants match response cards at the top of the screen to one of four stimulus cards at the bottom of the screen. The response cards were four different possible colours (blue, red, green and yellow), numbers (1 through 4) and four shapes (triangle, star, circle and cross). The participant is required to match the response card to the stimulus card by one of three rules (colour, number or shape) but is not told the rule. They must deduce the correct sorting category based on feedback of "correct" or "incorrect". After four correct matches the rule changes and the participant must then identify the new matching rule based on further feedback. Participants who perseverate would not be able to complete as many shifts as those who are able to shift quickly. The variable of interest for this study is total number of shifts.

Simon Task. This task was based on a modified Stroop Task designed by Hajcak, McDonald \& Simons (2003). Much like the Stroop task, the Simon task requires the respondent to inhibit a dominant response. The computer screen presents a series of red or green arrows oriented left, right or towards the top of the screen. Participants are directed to respond to the colour of the arrow by pressing the " $\mathrm{j}$ " key for green, and the "f" key for red requiring them to inhibit responding to the direction of the arrow. The incongruent trials are expected to incur the greatest level of errors (i.e. pressing the $\mathrm{j}$ key for green, even though the arrow is pointing left; Hajcak, McDonald \& Simons, 2003). There were a total of 200 trials with half congruent and half incongruent. The key variable of interest for this study is number of correct responses in the incongruent trials and the reaction time for these responses. Reaction time for correct incongruent trials was also recorded.

\section{Procedure}

As with study 1, participants were tested in small groups and were randomly assigned to either a rumination condition or a distraction condition. They first completed the baseline questionnaires followed by the induction and then the 3 cognitive tasks - CPT, WCST and the Simon task. Because the between group differences were the key comparison, the cognitive measures were presented in the same order for every participant. As with the first study, visual analogue scales assessed the participant's current mood between the tasks.

\section{Results and Discussion}

For this sample, the two experimental groups did not significantly differ on age $(\mathrm{t}=-.104)$ or baseline measures - RTS $(\mathrm{t}=$ $-1.103)$, PANAS-N $(\mathrm{t}=-.165)$ or PANAS-P $(\mathrm{t}=1.22)$. They also did not differ in their mood rating on the first visual analogue scale prior to the rumination or distraction induction. Correlations were completed between the baseline measures and were as predicted (Table 4).

An examination of the change in mood from before and after the induction shows that prior to induction, the two groups did not significantly differ $(\mathrm{t}=-.737)$ nor following the induction $(\mathrm{t}$ $=-1.776, \mathrm{p}=.08$ ) although this did approach significance with those in the rumination condition showing greater negative mood. To examine the prediction of post-induction mood, a hierarchical linear regression was completed.

Baseline mood was entered into the first step and induction condition and RTS score were entered into the second. Both baseline $\operatorname{mood}(\beta=-.235, p=.00)$ and RTS score $(\beta=-.630, p$ $=.00$ ) were significant predictors while induction condition was not.

Hierarchical regressions examined the prediction of continuous performance scores. As with Study 1, baseline mood was entered into the first step and RTS and condition in the second. Results replicate those found in the first study with ruminative thought style predicting errors of attention in the slow and fast blocks (Table 5), and baseline negative mood predicting errors of inhibition in the slow and fast blocks (Table 5). As before, induction condition was not a significant predictor.

The same statistical procedures were used for the two new measures of cognitive flexibility, the WCST and the Simon Task (Table 6). Ruminative thought styles predicted percentage correct on incongruent trials in the Simon task, and both ruminative thought style and baseline mood predicted reaction time for these trials. Further, both baseline mood and RTS score significantly predicted number of shifts in the WCST.

These findings support those obtained in Study 1 without the limitation of the random assignment anomaly. A ruminative thought style is related to impairments of attention and negative mood with impairments in inhibition in the continuous performance task. This suggests that for the continuous performance measure, mood and ruminative thinking are differentially related to attention and inhibition. However, this becomes less clear when we consider the findings for the Simon task and the WCST.

The Simon task was included as a measure of inhibition, however unlike the CPT, it is the ruminative thought style that significantly predicts performance on this task, with both mood and ruminative thought style predicting reaction time. Further,

Table 4.

Baseline correlations.

\begin{tabular}{ccccc}
\hline & PANAS-N & Mood 1 & M & SD \\
\hline RTS & $.476^{* *}$ & $-.749^{* *}$ & 89.58 & 19.62 \\
PANAS-N & & $-.539^{* *}$ & 21.39 & 7.21 \\
Mood 1 & & & .599 & .193 \\
\hline
\end{tabular}

Note: ${ }^{* *} p<.01$. 
Table 5.

Predicting cognitive performance by degree of cognitive load controlling for baseline mood.

\begin{tabular}{|c|c|c|c|c|c|c|}
\hline \multirow[b]{2}{*}{ High Cognitive Load } & \multicolumn{3}{|c|}{ False Hits (Inhibition) } & \multicolumn{3}{|c|}{ Correct Hits (Attention) } \\
\hline & B & SE B & $\beta$ & B & SE B & $\beta$ \\
\hline \multicolumn{7}{|l|}{ Step 1} \\
\hline Baseline Mood & .060 & .010 & $.480^{* *}$ & -.064 & .014 & $-.387^{* *}$ \\
\hline \multicolumn{7}{|l|}{ Step 2} \\
\hline Baseline Mood & .056 & .011 & $.451^{* *}$ & .001 & .010 & .004 \\
\hline RTS Score & .003 & .004 & .062 & -.051 & .004 & $-.820^{* *}$ \\
\hline Condition & .008 & .145 & .004 & .041 & .126 & .017 \\
\hline \multicolumn{7}{|l|}{ Medium Load } \\
\hline \multicolumn{7}{|l|}{ Step 1} \\
\hline Baseline Mood & -.018 & .008 & -.213 & -.018 & .011 & -.148 \\
\hline \multicolumn{7}{|l|}{ Step 2} \\
\hline Baseline Mood & -.012 & .008 & -.142 & -.018 & .012 & -.150 \\
\hline RTS Score & -.006 & .003 & -.175 & .001 & .004 & .017 \\
\hline Condition & -.177 & .109 & -.142 & .134 & .154 & .078 \\
\hline \multicolumn{7}{|l|}{ Low Load } \\
\hline \multicolumn{7}{|l|}{ Step 1} \\
\hline Baseline Mood & .025 & .008 & $.257^{* *}$ & -.069 & .015 & $-.387^{* *}$ \\
\hline \multicolumn{7}{|l|}{ Step 2} \\
\hline Baseline Mood & .018 & .009 & $.191^{+}$ & .003 & .010 & .020 \\
\hline RTS Score & .005 & .004 & .137 & -.056 & .004 & $-.850^{* *}$ \\
\hline Condition & .000 & .122 & .000 & .047 & .127 & .018 \\
\hline
\end{tabular}

Note: ${ }^{*} p<.05 ;{ }^{* *} p<.01 ;{ }^{+} p=.055$.

both mood and RTS score predict performance on the WCST. These tasks may draw upon different, if overlapping abilities in executive function.

\section{General Discussion}

As hypothesised, a ruminative thought style predicted cognitive impairments when cognitive load was low, and the combination of rumination and off-task thoughts surpassed the main effect in predictive ability. This same relationship was found when cognitive load was high. When task demands are low, cognitive resources remain available for intrusive thoughts which then detract from performance on the task at hand (Teasdale et al., 1995) but there comes a point where the task demands become too great, and the influence of ruminative thought returns (Lybomirsky, Kasri, \& Zehm, 2003). This is of considerable clinical relevance when treating clients with depressed mood and a tendency to ruminate. Depression can lead to social withdrawal, and isolation to the point where the individual may spend days or even weeks with very little external stimulation (Watson \& Andrews, 2002). In this situation, the cognitive load would be minimal, leaving room for intrusive ruminative thoughts to thrive. Watson and Andrews (2002) suggest that this withdrawal and minimization of external distractions is an adaptive behaviour to help focus the individual on finding solutions to their stressors. However, this adaptivebehaviour may become maladaptive when paired with a dispo
Table 6.

Predicting simon and wisconsin card sort task scores.

\begin{tabular}{|c|c|c|c|}
\hline Simon Task Incongruent & B & SE B & $\beta$ \\
\hline \multicolumn{4}{|l|}{ Step 1} \\
\hline Baseline Mood & -.143 & .176 & -.073 \\
\hline \multicolumn{4}{|l|}{ Step 2} \\
\hline Baseline Mood & .171 & .191 & .087 \\
\hline RTS Score & -.263 & .071 & $-.359^{* *}$ \\
\hline Induction Condition & -3.831 & 2.453 & -.135 \\
\hline \multicolumn{4}{|c|}{ Simon Task Reaction Time } \\
\hline \multicolumn{4}{|l|}{ Step 1} \\
\hline Baseline Mood & .519 & .813 & .057 \\
\hline \multicolumn{4}{|l|}{ Step 2} \\
\hline Baseline Mood & 1.895 & .892 & $.209^{*}$ \\
\hline RTS Score & -1.119 & .334 & $-.332^{* *}$ \\
\hline Induction Condition & -9.933 & 11.470 & -.076 \\
\hline \multicolumn{4}{|c|}{ WCST Number of Shifts } \\
\hline \multicolumn{4}{|l|}{ Step 1} \\
\hline Baseline Mood & -.051 & .038 & -.117 \\
\hline \multicolumn{4}{|l|}{ Step 2} \\
\hline Baseline Mood & -.121 & .042 & $-.280^{* *}$ \\
\hline RTS Score & .056 & .016 & $.349^{* *}$ \\
\hline Induction Condition & .230 & .540 & .037 \\
\hline
\end{tabular}

Note: ${ }^{*} p<.05 ;{ }^{* *} p<.01$.

sition towards a ruminative style of thinking. At the other end, numerous stressors, may increase cognitive load to a point where mood and rumination again reduce the ability to inhibit intrusive thoughts and to maintain attention elsewhere, such as on problem solving strategies that might otherwise resolve the stressors.

Koster, De Lissnyder, Derakshan and De Raedt (2011) propose the impaired disengagement hypothesis and describe a circular process of stress, ruminative thinking, negative mood, further ruminative thinking and so on. If one is predisposed to a ruminative style of thinking, as a result of or concomitant to an individual difference in executive function, the experience of this stressor may indeed trigger this maladaptive cycle, especially if the stressor consumes considerable cognitive resources, or compels the individual to withdraw thereby eliminating external demands or distracters. In these two situations, the individual would not be able to maintain attention elsewhere such as identifying strengths and resources or on solution generation.

A key consideration in this process is the apparent differential influences of mood and ruminative thinking. Intuitively, rumination would seem to be a combination of inability to inhibit ruminative thoughts and the inability to maintain attention elsewhere. However current findings suggest that negative mood is the phenomenon related to the inability to inhibit and this has been supported in previous research (Lau et al., 2007). Rogers et al. (2004) describe mixed results from numerous studies examining negative mood and executive dysfunction, but state that most studies have reported deficits in set-shifting. Much of the previous research has measured set-shifting using 
the Wisconsin Card Sorting Task, which does not easily differentiate between the inhibition of previous set and the maintaining of attention on the new set. Executive functions are a complex set of abilities and reside in a number of brain structures limiting the ability to examine them in isolation (Rogers et al., 2004). In reality, attempts to differentiate executive functions may be as artificial as trying to separate depression and rumination since the interaction between these functions is inevitable. However, it does suggest that the amelioration of depressed mood may not necessarily influence the tendency to ruminate, and this lingering behaviour, may pose a vulnerability to future depressive episodes.

Finally, it is important to note that the experimental inductions used in these experiments did not produce differences in mood or cognitive performance and this makes perfect sense given the theory on which they were created (Lyubomirsky \& Nolen-Hoeksema, 1995; Nolen-Hoeksema, Parker, \& Larson, 1994). In the response style theory, individuals ruminate in response to existing depressed mood. Asking participants to spend time focusing on themselves, how they feel and the possible causes and consequences of those feelings would not produce consistent outcomes if those individuals are not all experiencing negative mood. Viewing rumination as a style of thinking is not in conflict with the response style theory, but simply a different perspective that examines the process of rumination. New induction procedures that mimic the process of rumination, independent of content and mood, are needed to undertake an experimental examination and to establish causal direction. Current findings show that a ruminative style of thinking predicts subsequent performance, and it may be that rumination impairs executive function. However, it seems more intuitive that structure leads function, and therefore individual differences in executive function dispose some individuals to a ruminative style of thinking. Until it is possible to manipulate one and to observe changes in the other, the causal direction in this relationship will remain unknown. This area of research has many more questions than answers, but in time the relationship between ruminative thinking, executive function and mood will be clarified and provide further guidance in treating depressed mood.

\section{REFERENCES}

Antrobus, J. S. (1968). Information theory and stimulus-independent thought. British Journal of Psychology, 59, 423-430.

doi:10.1111/j.2044-8295.1968.tb01157.x

Austin, M., Mitchell, P., \& Goodwin, G. M. (2001). Cognitive deficits in depression. British Journal of Psychiatry, 178, 200-206. doi:10.1192/bjp.178.3.200

Brinker, J. K., \& Dozois, D. J. A. (2009). Ruminative thought style and depressed mood. Journal of Clinical Psychology, 65, 1-19. doi: $10.1002 /$ jclp. 20542

Carson, S. H., Peterson, J. B., \& Higgins, D. M. (2003). Decreased latent inhibition is associated with increased creative achievement in high functioning individuals. Journal of Personality and Social Psychology, 85, 499-506. doi:10.1037/0022-3514.85.3.499

Davis, R. N., \& Nolen-Hoeksema, S. (2000). Cognitive inflexibility among ruminators and non-ruminators. Cognitive Therapy and Research, 24, 699-711. doi:10.1023/A:1005591412406

Grant, D. A., \& Berg, E. A. (1948). A behavioral analysis of reinforcement and ease of shifting to new responses in a Weigel-type cardsorting problem. Journal of Experimental Psychology, 38, 404-411. doi: $10.1037 / \mathrm{h} 0059831$

Hajcak, G., McDonald, N., \& Simons, R. F. (2003). To err is autonomic: Error-related brain potentials, ANS activity, and post-error compen- satory behavior. Psychophysiology, 40, 895-903. doi:10.1111/1469-8986.00107

Hertel, P. T. (1997). On the contributions of deficient cognitive control to memory impairments in depression. Cognition \& Emotion, 11, 569-584. doi:10.1080/026999397379890a

Joorman, J., Yoon, K. L., \& Zetsche, U. (2007). Cognitive inhibition in depression. Applied and Preventative Psychology, 12, 128-139. doi:10.1016/j.appsy.2007.09.002

Koster, E. H. W., De Lissnyder, E., Derakshan, N., \& De Raedt, R. (2001). Understanding depressive rumination from a cognitive science perspective: The impaired disengagement hypothesis. Clinical Psychology Review, 31, 138-145. doi:10.1016/j.cpr.2010.08.005

Lau, M., Christensen, B. K., Hawley, L. L., Gemar, M. S., \& Segal, Z. V. (2007). Inhibitory deficits for negative information in persons with major depressive disorder. Psychological Medicine, 37, 1249 1259. doi: $10.1017 / \mathrm{S} 0033291707000530$

Lo, B. C. Y., Lau, S., Cheung, S., \& Allen, N. B. (2012). The impact of rumination on internal attention switching. Cognition and Emotion, 26, 209-203. doi:10.1080/02699931.2011.574997

Lyubomirsky, S., Kasri, F., \& Zehm, K. (2003). Dysphoric rumination impairs concentration on academic tasks. Cognitive Therapy and Research, 27, 309-330. doi:10.1023/A:1023918517378

Lyubomirsky, S., \& Nolen-Hoeksema, S. (1993). Self-perpetuating properties of dysphoric rumination. Journal of Personality and Social Psychology, 65, 339-349. doi:10.1037/0022-3514.65.2.339

Lyubomirsky, S., \& Nolen-Hoeksema, S. (1995). Effects of self-focused rumination on negative thinking and interpersonal problem solving. Journal of Personality and Social Psychology, 69, 176-190. doi:10.1037/0022-3514.69.1.176

Martin, L. L., \& Tesser, A. (1996). Some ruminative thoughts. In R. S. Wyer Jr. (Ed.), Ruminative thoughts (pp. 1-47). Hillsdale, NJ: Erlbaum.

Miyake, A., Friedman, N. P., Emerson, M. J., Witzki, A. H., Howerter, A., \& Wager, T. D. (2000). The unity and diversity of executive functions and their contribution to complex "frontal-lobe" tasks: A latent variable analysis. Cognitive Psychology, 41, 49-100. doi:10.1006/cogp.1999.0734

Mor, N., \& Winquist, J. (2002). Self-focused attention and negative affect: A meta-analysis. Psychological Bulletin, 128, 638-662. doi:10.1037/0033-2909.128.4.638

Nolen-Hoeksema, S. Parker, L., \& Larson, J. (1994). Ruminative coping with depressed mood following loss. Journal of Personality and Social Psychology, 67, 92-104. doi:10.1037/0022-3514.67.1.92

Nolen-Hoeksema, S., Wisco, B. E., \& Lyubomirsky, S. (2008). Rethinking rumination. Perspectives on Psychological Science, 3, 400424. doi:10.1111/j.1745-6924.2008.00088.x

Papageorgiou, C., \& Siegle, G. J. (2003). Rumination and depression: Advances in theory and research. Cognitive Therapy and Research, 27, 243-245. doi:10.1023/A:1023918331490

Rimes, K. A., \& Watkins, E. (2005). The effects of self-focused rumination on global negative self-judgements in depression. Behaviour Research and Therapy, 43, 1673-1681.

doi:10.1016/j.brat.2004.12.002

Rogers, M. A., Kasai, K., Matsuo, K., Fukuda, R., Iwanami, A., Nakagome, K., Fukuda, M., \& Kato, N. (2004). Executive and prefrontal dysfuction in unipolar depression: A review of neuropsychological and imaging evidence. Neuroscience Research, 50, 1-11. doi:10.1016/j.neures.2004.05.003

Teasdale, J. D., Dritschel, B. H., Taylor, M. J., Proctor, L., Lloyd, C. A., Nimmo-Smith, I., \& Baddeley, A. D. (1995). Stimulus-independent thought depends on central executive resources. Memory \& Cognition, 23, 551-559. doi:10.3758/BF03197257

Verhaeghen, P., Joorman, J., \& Khan, R. (2005). Why we sing the blues: The relation between self-reflective rumination, mood, and creativity. Emotion, 5, 226-232. doi:10.1037/1528-3542.5.2.226

Watkins, E., \& Brown, R. G. (2002). Rumination and executive function in depression: An experimental study. Journal of Neurology, Neurosurgery and Psychiatry, 72, 400-402. doi:10.1136/jnnp.72.3.400

Watkins, E., \& Moulds, M., (2005). Distinct modes of ruminative selffocus: Impact of abstract versus concrete rumination on problem solv- 


\section{J. K. BRINKER ET AL.}

ing in depression. Emotion, 5, 319-328.

doi: $10.1037 / 1528-3542.5 .3 .319$

Watson, D., \& Clark, L. A. (1994). Manual for the positive and negative affect schedule-expanded form. Iowa City, IA: The University of Iowa. http://www.psychology.uiowa.edu/faculty/clark/panas-x.pdf

Watson, P. J., \& Andrews, P. W. (2002). Toward a revised evolutionary adaptionist analysis of depression: The social navigation hypothesis. Journal of Affective Disorders, 72, 1-14.
doi:10.1016/S0165-0327(01)00459-1

Whitmer, A. J., \& Banich, M. T. (2007). Inhibition versus switching deficits in different forms of rumination. Psychological Science, 18, 546-553. doi:10.1111/j.1467-9280.2007.01936.x

Williams, P. G., Suchy, Y., \& Rau, H. K. (2009) Individual differences in executive functioning: Implications for stress regulation. Annals of Behavioral Medicine, 37, 126-140. doi:10.1007/s12160-009-9100-0 